\title{
Time of Flight Backscatter and Secondary Ion Spectrometry in a Helium Ion Microscope
}

\author{
Nico Klingner ${ }^{1}$, Gregor Hlawacek ${ }^{1}$, Rene Heller ${ }^{1}$, Johannes von Borany ${ }^{1}$ and Stefan Facsko ${ }^{1}$ \\ 1. Institute for Ion Beam Physics and Materials Research, Helmholtz-Zentrum Dresden-Rossendorf, \\ Bautzner Landstr. 400, Dresden, Germany.
}

Helium Ion Microscopy (HIM) utilizes a Gas Field Ion Source (GFIS) to create a Helium or Neon ion beam with a diameter better than $0.5 \mathrm{~nm}$ and $1.8 \mathrm{~nm}$, respectively. The method is well known for its high resolution imaging and nano-fabrication capabilities which it is able to provide not only for conducting but also insulating samples without the need for a conductive coating. The latter specimens are typically found in the fields of biosciences, MEMS/NEMS technology, catalyst research and many others. The availability of $\mathrm{He}$ and $\mathrm{Ne}$ ions with either low or moderate sputter yields, allow precision direct write nano-structuring with a precision below $10 \mathrm{~nm}$ in the HIM [1, 2].

However, the existing GFIS based focused ion beam (FIB) tools suffer from the lack of a well integrated analytic method that can enrich the highly detailed morphological images with materials contrast. While the technologyHIM technology is relatively young several efforts have been made to add such an analytic capability to the technique. So far, ionoluminescence $[1,3]$, backscattering spectrometry (BS) $[1,4,5]$, and secondary ion mass spectrometry (SIMS) using a magnetic sector [6] or time of flight (TOF) setup have been demonstrated [4].

After a brief introduction to HIM itself and a summary of the existing approaches I will focus on our own time of flight based analytic approaches. TOF-HIM is enabled by using a fast blanking electronics to chop the primary beam into pulses with a minimal length of only $20 \mathrm{~ns}$. In combination with an MCPmultichannel-plate based stop detector this enables TOF backscatter spectrometry (TOF-BS) using $\mathrm{He}$ ions at an energy of only $30 \mathrm{keV}$. The achieved lateral resolution is $54 \mathrm{~nm}$ and represents a world record for spatially resolved backscattering spectrometry. The achieved energy resolution has been measured to be of $1.5 \mathrm{keV}(5 \%)$. This is sufficient to separate most of the elements (see fig. 1) and allows the detection of thin surface layers formed from heavy elements. The results will be compared to the theoretical achievablereachable lateral and energy resolution and the limiting experimental and physical constraints of this approach will be reviewed.

Finally In the last part I will first present TOF-SIMS results obtained with a very simple experimental configuration will be presented. Based on the findings obtained with this poor man's version of TOFSIMS setup a dedicated extraction optics for secondary ions has been designed and tested. This revised e setup can be operated in point and shoot mode to obtain high resolution SIMS data or in imaging mode to obtain element maps of the specimen surface. First experiments revealed a very high relative transmission of up to $76 \%$ which is crucial to collect enough signal from nanoparticles prior to their complete removal by ion sputtering. For $\mathrm{m} / \mathrm{q} \leq 80 \mathrm{u}$ a $\mathrm{Dm} \leq 0.3 \mathrm{u}$ has been achieved. This is sufficient for many life science applications that rely on the isotope identification of light elements (e.g.: C, N). The lateral resolution of $8 \mathrm{~nm}$ has been evaluated using the knife edge method and a $75 \% / 25 \%$ criterion andwhich represents a world record for spatially resolved secondary ion mass spectrometry. 
References:

[1] G. Hlawacek et al, J. Vac. Sci. Technol. B Microelectron. Nanom. Struct. 32 (2014), p. 020801. [2] G. Hlawacek et al, in "Helium Ion Microscopy", ed. G. Hlawacek and A. Gölzhäuser, (Springer International).

[3] V. Veligura et al, J. Lumin. 157 (2015), p. 321.

[4] N. Klingner et al, Ultramicroscopy 162 (2016), p. 91.

[5] R. Heller, N. Klingner, G. Hlawacek in "Helium Ion Microsc.", ed. G. Hlawacek and A. Gölzhäuser, (Springer International).

[6] T. Wirtz, D. Dowsett, and P. Philipp in "Helium Ion Microsc.", ed. G. Hlawacek and A. Gölzhäuser, (Springer International).
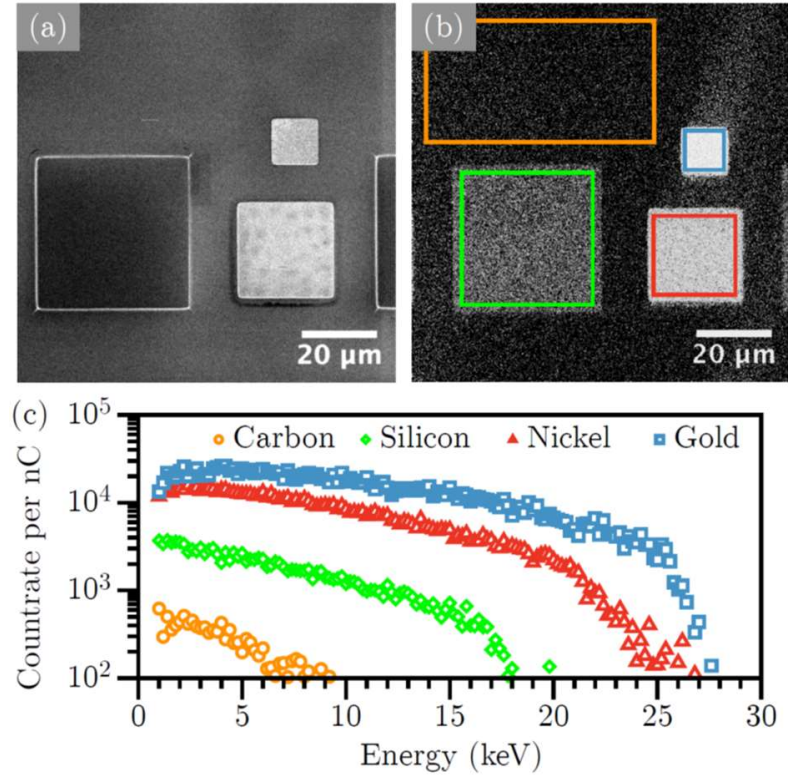

Figure 1. SE (a) and TOF-BS image (b) and spectra (c). The individual patches on the test sample can be identified by their difference in TOF (b). From the high energy edge of the spatially resolved spectra (c) the dominant elements in the investigated area can be identified.

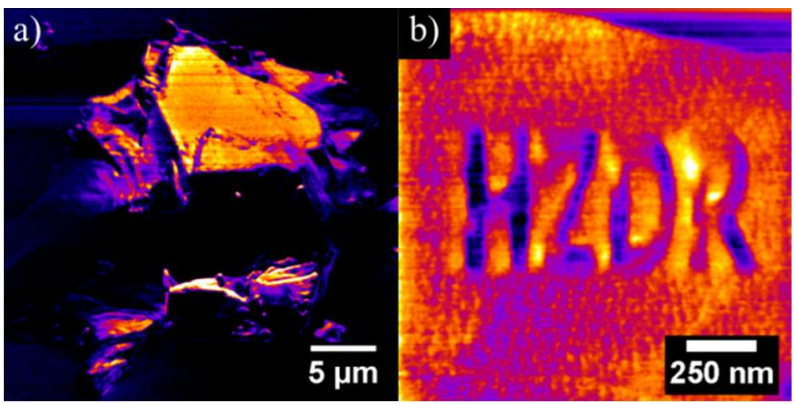

Figure 2. (a) $\mathrm{SIMS}^{+}$image of a rock salt crystal on $\mathrm{SiO}_{2}$. (b) $\mathrm{Na}^{+} \mathrm{SIMS}^{+}$image of the $\mathrm{Ne}$ ion beam milled letters $\mathrm{HZDR}$. The contrast arises as the $\mathrm{NaCl}$ has been partially removed by the $\mathrm{Ne}$ milling. 